\title{
Subcutaneous tumor seeding after biopsy in gliomatosis cerebri
}

\author{
Dennis R. Buis · Paul van der Valk • \\ Philip C. De Witt Hamer
}

Received: 17 April 2011 / Accepted: 30 July 2011/Published online: 12 August 2011

(C) The Author(s) 2011. This article is published with open access at Springerlink.com

\begin{abstract}
We observed a patient with subcutaneous seeding from gliomatosis cerebri with a low-grade histopathology. A 33-year-old woman with neurofibromatosis type 1 presented with progressive headache, diplopia, dysphagia, and a rightward instability. On neurological examination dysarthria, gait ataxia, and left-sided central facial and hypoglossal palsies were determined. MRI of the brain demonstrated diffuse, infiltrative non-enhancing lesions in the pons, both cerebellar hemispheres, the parahippocampal gyrus, and the thalamus. A stereotactic biopsy demonstrated an astrocytoma WHO grade 2. These characteristics confirmed gliomatosis cerebri. Three months later, the patient presented with hydrocephalus and a subcutaneous swelling directly underneath the surgical scar. The subcutaneous swelling was removed and the hydrocephalus was treated by ventriculoperitoneal shunting. Histopathological examination confirmed a subcutaneous manifestation of low-grade oligoastrocytoma. Gliomatosis cerebri with low-grade histology can seed subcutaneously.
\end{abstract}

Keywords Tumor seeding - Stereotactic biopsy . Glioma · Pathology

D. R. Buis $(\square)$ · P. C. De Witt Hamer

Department of Neurosurgery,

ZH 2F005, Neurosurgical Center Amsterdam,

VU University Medical Center, PO Box 7057,

1007 MB Amsterdam, The Netherlands

e-mail: drbuis@gmail.com

P. van der Valk

Department of Neuropathology, VU University Medical Center,

Amsterdam, The Netherlands

\section{Case report}

A 33-year-old female with neurofibromatosis type 1 presented with progressive headache since one year. Daily nausea and vomiting progressed since six months. Diplopia, dysphagia, and right-ward instability were present since one month. On neurological examination dysarthria and gait ataxia were confirmed. Left-sided central facial and hypoglossal palsies were noted. She had diplopia, when looking to the right with a grade 1 nystagmus and a central hypesthesia of the right side of the face. Babinski's sign and right-sided hyperreflexia were noted. An MRI of the brain demonstrated a diffuse infiltrative, non-enhancing lesion reaching from the pons to both cerebellar hemispheres, and extending to the basal ganglia and thalamus. This resulted in bilateral diffuse swelling of the thalamus, hypothalamus, and basal ganglia. Additionally, there were manifestations around the right frontal lobe, the insula, amygdala, and the parahippocampal gyrus. These radiological characteristics were compatible with gliomatosis cerebri (Fig. 1).

To establish a histopathological diagnosis, she had a frameless stereotactic needle biopsy of the right mesiotemporal region with a Sedan side cutting needle through a regular burrhole (Fig. 2). A total number of three biopsies at the same location were taken. The small dura opening was covered with a Novacol pad (Taureon, Rijswijk, The Netherlands), but left otherwise unclosed and unsealed. The skin and subcutaneous tissue, and temporal fascia were closed in layers.

The histopathological examination demonstrated irregular cells with a clear atypia of the GFAP positive staining cells (Fig. 3). Given the relative paucity of these cells and their diffuse spread throughout the biopsy, a diagnosis of gliomatosis of the astrocytic subtype (WHO grade 2) was 

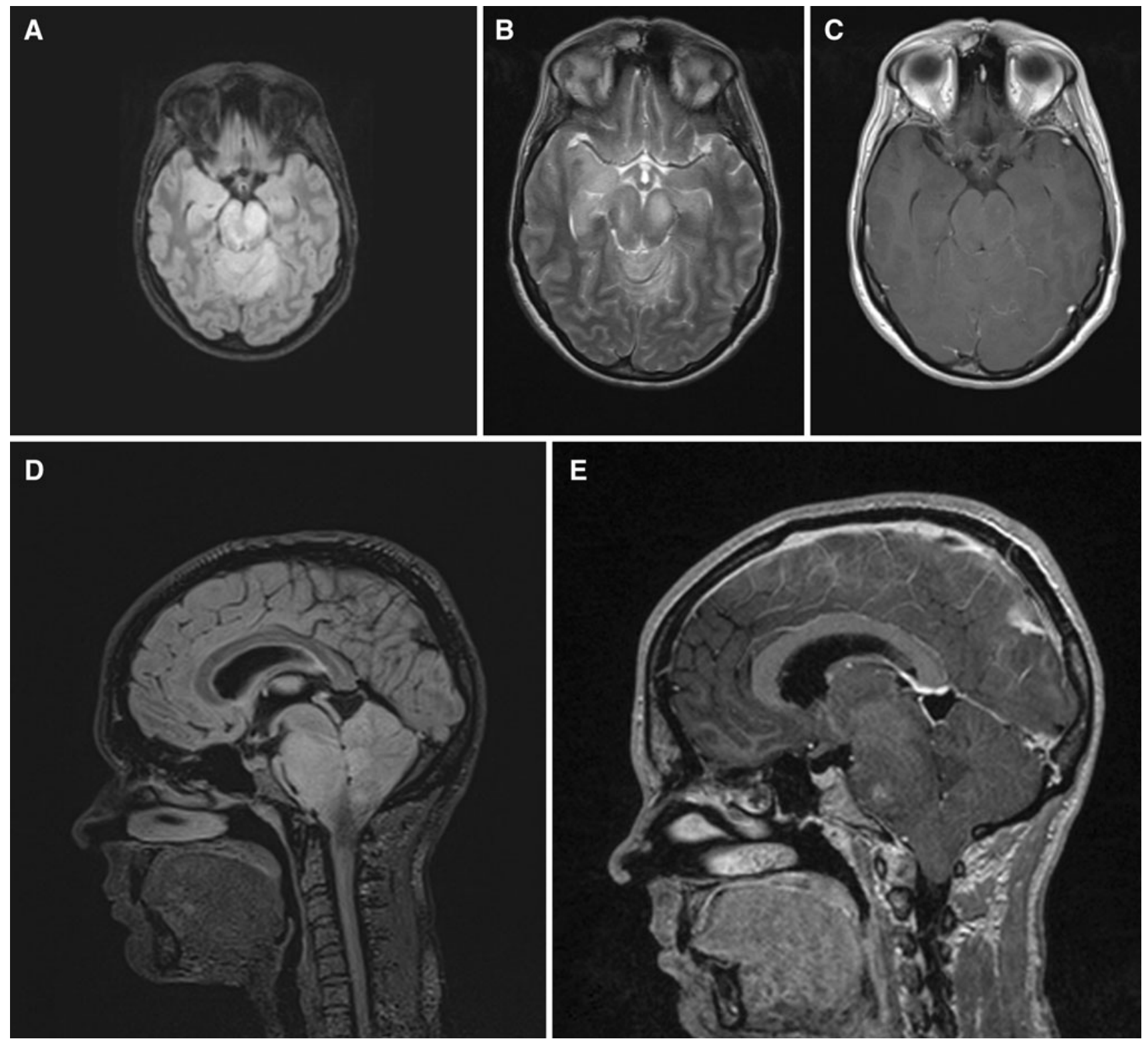

Fig. 1 The diffusely swollen mesencephalon, uncus and parahippocampal gyrus on FLAIR (A) and $\mathrm{T}_{2}$ MRI-sequences (B), as well as the absence of gadolinium enhancement $(\mathbf{C}$ and $\mathbf{E})$ is compatible with

made. Due to profound invasion, resulting in too extensive radiation fields, radiotherapy was refrained and temozolomide monotherapy was initiated. Her headaches improved with Dexamethasone. However, three months later, the headaches progressed, accompanied by a subcutaneous swelling at the incision, due to which the patient was unable to wear her glasses (Fig. 4). CT-imaging demonstrated triventricular hydrocephalus. In order to relief her headaches, she was given a ventriculoperitoneal shunt and the swelling was removed completely through a re-incision.

the radiological criteria for gliomatosis cerebri. There is diffuse enlargement of the pons (D)

Histopathological examination of the subcutaneous swelling demonstrated skin and subcutaneous tissue invaded by glial tumor, which was diagnosed to be oligoastrocytoma, as the sample was dominated by oligodendrocytes with a clear cytoplasm and slightly irregular nuclei, but also a component of large, multinucleated astrocytic cells, which were morphologically similar to the biopsy sample (Fig. 5).

After placement of the shunt, her headaches improved, but the intracranial tumor progressed clinically during temozolomide, which was subsequently stopped. Although, 

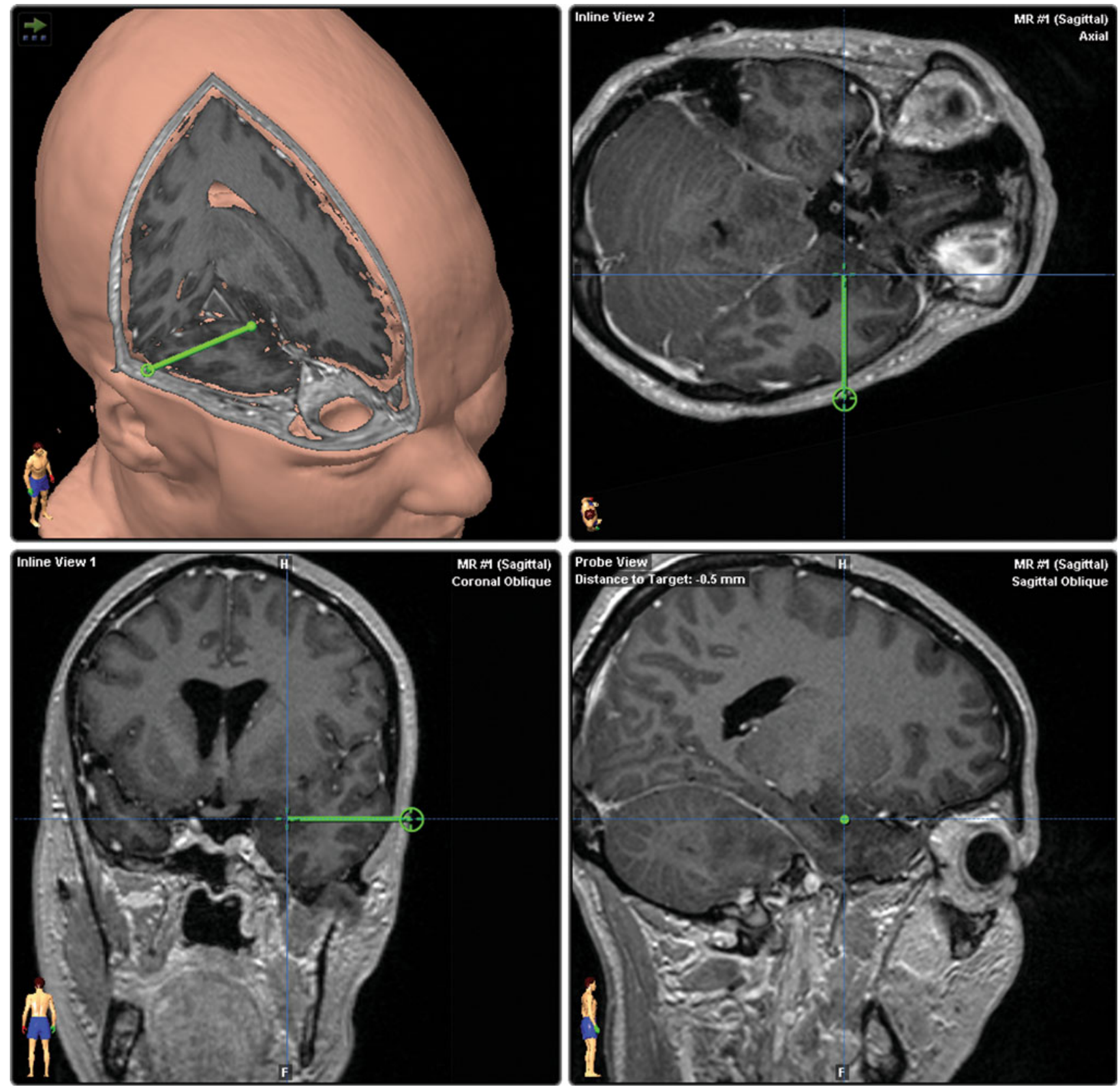

Fig. 2 A needle biopsy was taken from tumor in the right mesiotemporal region (green tract)

our patient was offered second line chemotherapy, she refused further treatment and died shortly after.

\section{Discussion}

Approximately $20 \%$ of patients with neurofibromatosis type 1 develop low-grade gliomas [1], but gliomatosis cerebri is rare, even in association with neurofibromatosis [2]. Gliomatosis cerebri is a glioma with a diffuse, usually astrocytic growth pattern, that widely invades the brain involving at least three cerebral lobes, and usually with bilateral involvement of the cerebral hemispheres, deep gray matter, brainstem or cerebellum [3]. Although, mitotic activity is generally low, and anaplastic characteristics, such as microvascular proliferation and necrosis are generally absent at presentation [4-6], its biologic behaviour corresponds to a WHO grade 3 tumor in the majority of cases [3].

Subcutaneous metastasis from gliomas are rare [7]. However, in high-grade glioma, remote cutaneous 
Fig. 3 Initial brain biopsy. The left hand panel shows hematoxilin-eosin-stained section of cortical tissue with slight increase in cellularity (objective $\times 2.5$ ). The cells are somewhat irregular, as is better appreciated in the right hand panel, a GFAP staining for astrocytic cells (objective $\times 40$ ), showing clear atypia of the positive-staining cells. Given the relative paucity of these cells and their diffuse spread throughout the biopsy a histopathological diagnosis of gliomatosis was made
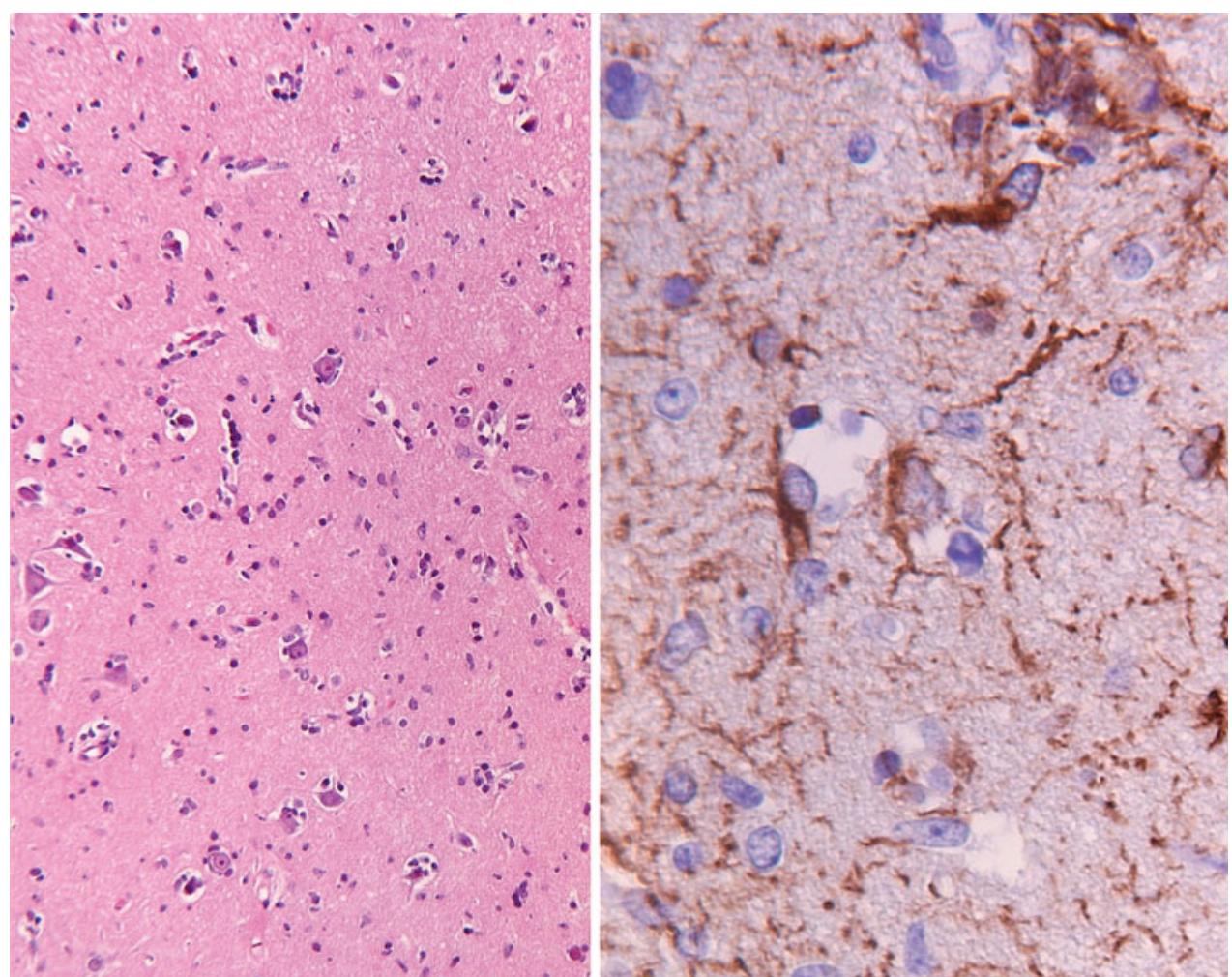

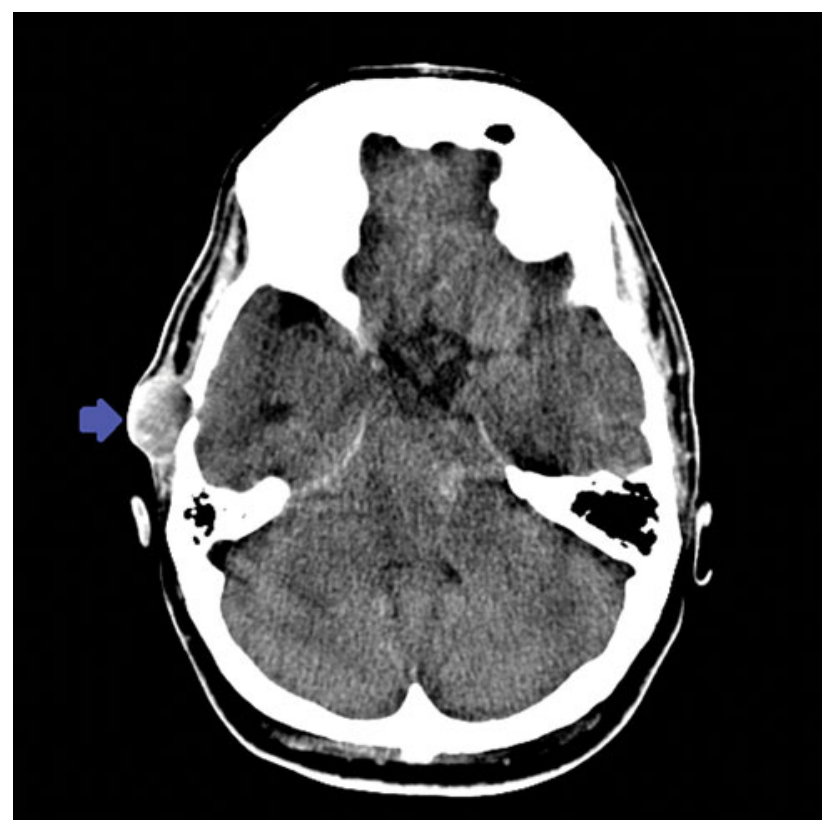

Fig. 4 The subcutaneous lesion (blue arrow), in the biopsy tract is not continuous with the intracranial compartment. The hyperdensity in the pons is thought to be a tumor related bleeding, which was probably not biopsy related metastasis [8-10], or metastatic seeding through the stereotactic biopsy tract [11-14] have been reported. The presumed mechanism includes seeding of tumor cells during surgery [13], especially, when the biopsy tract was not included in the irradiated volume [14]. Seeding of tumor in the biopsy tract has not been reported in gliomatosis cerebri or low-grade glioma. Alternatively, the glioma grading may have been underestimated due to biopsy sampling error.

We postulate that in the presented case, tumor cells were spilled and implanted during removal of the needle, as the intra- and extracranial tumor components were not continuous on CT imaging and at visual inspection during removal of the lesion. Therefore our observation demonstrates that gliomatosis cerebri with low-grade histopathology has the potential to seed subcutaneously. This observation warrants several practical advises. First, close inspection for tumor fragments in the surgical field during biopsy seems to be required. Second, extensive rinsing of the surgical field may facilitate removal of tumor elements that cannot be detected by inspection. Third, tight dural sealing or closure to avoid secondary subcutaneous tumor spill is advisable. 
Fig. 5 Resection specimen of skin and subcutaneous tissues. The inset in the top left hand corner shows a low-power view, showing epidermis and dermis. In the larger figure, the image is dominated by oligodendrocytes with a clear cytoplasm and slightly irregular nuclei; in addition, there is a component of large, multinucleated astrocytic cells, warranting the diagnosis of oligoastrocytoma in this specimen. Both figures are hematoxilin-eosin stained. (The inset is a $\times 1.25$ objective, the large figure $a \times 40$ objective)

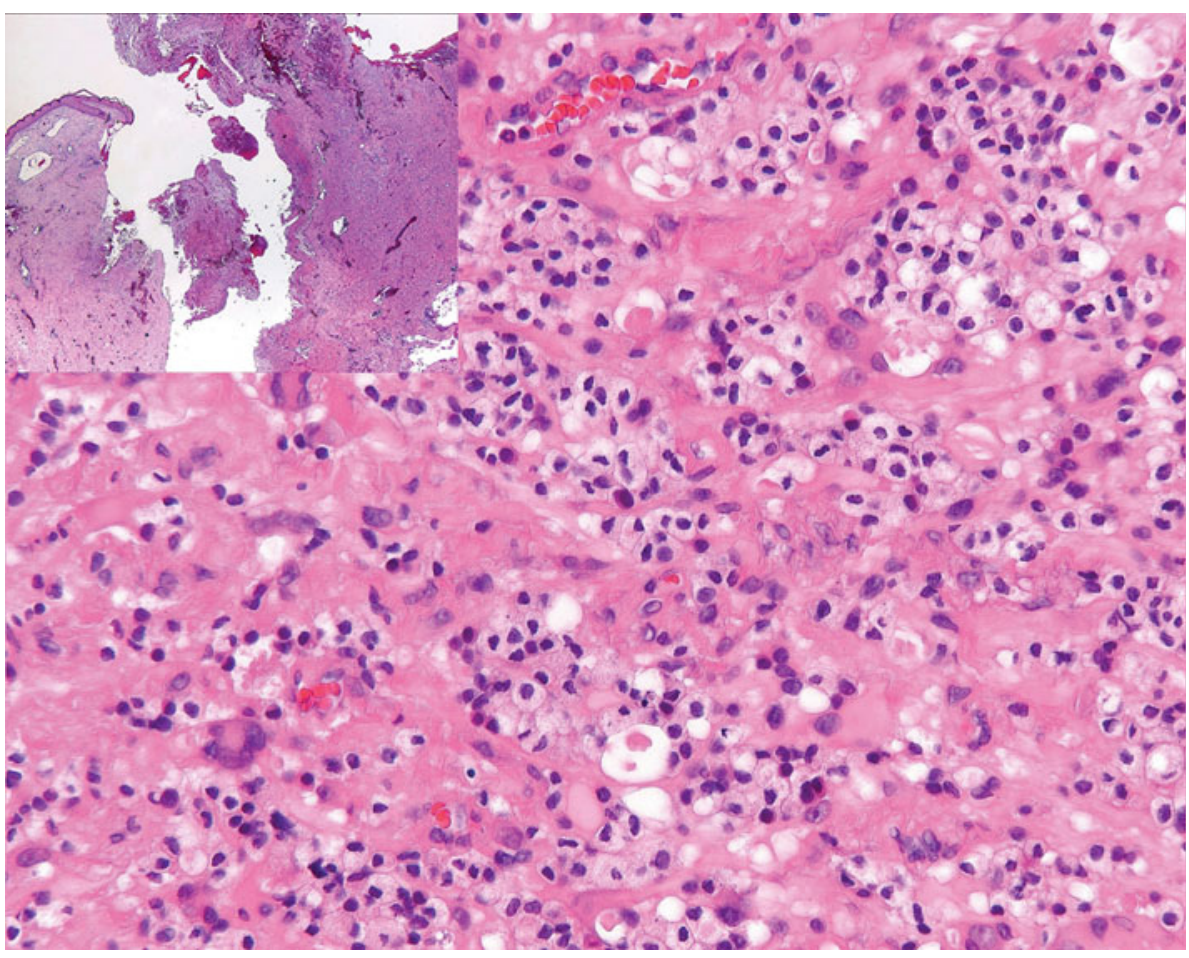

Open Access This article is distributed under the terms of the Creative Commons Attribution Noncommercial License which permits any noncommercial use, distribution, and reproduction in any medium, provided the original author(s) and source are credited.

\section{References}

1. Rosenfeld A, Listernick R, Charrow J, Goldman S (2010) Neurofibromatosis type 1 and high-grade tumors of the central nervous system. Childs Nerv Syst 26:663-667

2. Perilongo G, Garre ML, Giangaspero F (2003) Low-grade gliomas and leptomeningeal dissemination: a poorly understood phenomenon. Childs Nerv Syst 19:197-203

3. Fuller GN, Kros JM (2007) Gliomatosis cerebri. In: Louis DN, Ohgaki H, Wiestler OD, Cavanee WK (eds) WHO Classification of Tumours of the Central Nervous System. International Agency for Research on Cancer (IARC), Lyon, pp 50-52

4. Claes A, Idema AJ, Wesseling P (2007) Diffuse glioma growth: a guerilla war. Acta Neuropathol 114:443-458

5. Demuth T, Berens ME (2004) Molecular mechanisms of glioma cell migration and invasion. J Neurooncol 70:217-228

6. Chen X, Dai J, Jiang T (2010) Supratentorial WHO grade II glioma invasion: a morphologic study using sequential conventional MRI. Br J Neurosurg 24:196-201

7. Pedersen PH, Rucklidge GJ, MOrk SJ, Terzis AJ, Engebraaten O, Lund-Johansen M, Backlund EO, Laerum OD, Bjerkvig R (1994)
Leptomeningeal tissue: a barrier against brain tumor cell invasion. J Natl Cancer Inst 86:1593-1599

8. Miliaras G, Tsitsopoulos PP, Markoula S, Kyritsis A, Polyzoidis KS, Malamou-Mitsi V (2009) Multifocal glioblastoma with remote cutaneous metastasis: a case report and review of the literature. Cen Eur Neurosurg 70:39-42

9. Figueroa P, Lupton JR, Remington T, Olding M, Jones RV, Sekhar LN, Sulica VI (2002) Cutaneous metastasis from an intracranial glioblastoma multiforme. J Am Acad Dermatol 46:297-300

10. Mentrikoski M, Johnson MD, Korones DN, Scott GA (2008) Glioblastoma multiforme in skin: a report of 2 cases and review of the literature. J Am Acad Dermatol 30(4):381-384

11. Steinmetz M, Barnett G, Kim BS, Chidel M, Suh J (2001) Metastatic seeding of the stereotactic biopsy tract in glioblastoma multiforme: case report and review of the literature. J Neurooncol 55:167-171

12. Bouillot-Eimer S, Loiseau H, Vital A (2005) Subcutaneous tumoral seeding from a glioblastoma following stereotactic biopsy: case report and review of the literature. Clin Neuropathol 24:247-251

13. Aichholzer M, Mazal PR, Haberler C, Dietrich W, Bertalanffy A, Roessler K, Ungersboeck K (2001) Epidural metastasis of a glioblastoma after stereotactic biopsy: case report. Minim Invasive Neurosurg 44:175-177

14. Pierallini A, Caramia F, Piattella MC, Pantano P, Santoro A, Di Stefano D, Bozzao L (1999) Metastasis along the stereotactic biopsy trajectory in glioblastoma multiforme. Acta Neurochir 141:1011-1012 